Revista Signos

2008, 41(67)

203-229

\title{
Comprensión de textos académicos escritos en inglés: Relación entre nivel de logro y variables involucradas*
}

\author{
Romualdo Ibáñez \\ Pontificia Universidad Católica de Valparaíso \\ Chile
}

Resumen: Hoy en día es esencial para los alumnos universitarios poseer habilidades psicodiscursivas que les permitan enfrentar textos disciplinares en forma exitosa. No obstante ello, en países donde el inglés es considerado una lengua extranjera, lo anteriormente señalado no es suficiente. Debido, precisamente, a que el inglés se ha convertido en una lengua franca, no solo en términos de negocios internacionales y desarrollo tecnológico, sino que también en lo referente a la investigación y la educación, en países como Chile, donde esta lengua no es la materna, los alumnos universitarios también deben desarrollar la habilidad para comprender textos disciplinares escritos en inglés. Considerando tal problemática, en esta investigación estudiamos el proceso de comprensión llevado a cabo por alumnos universitarios al enfrentar textos disciplinares escritos en inglés. Los estudiantes $(\mathrm{n}=84)$ pertenecen a la carrera de Química Industrial de la Pontificia Universidad Católica de Valparaíso. Nos focalizamos en el nivel de comprensión alcanzado por estos alumnos al enfrentar textos disciplinares escritos en inglés y también en la forma en que dicho nivel de comprensión es determinado por el nivel de dominio del inglés de los alumnos, su grado de inserción en la comunidad disciplinar y su habilidad para comprender textos en lengua materna. Para ello, llevamos a cabo un estudio correlacional, el que mostró que la relación entre el nivel de comprensión que los alumnos alcanzaron y cada una de las variables consideradas no representa una correlación positiva fuerte. Un hallazgo interesante es que, a partir de los análisis realizados, también, se determinó que la variable con mayor incidencia en el nivel de comprensión del texto académico escrito en inglés correspondió a la variable nivel de dominio del inglés, seguida de la variable inserción disciplinar y, por último, a la variable habilidad de comprensión en lengua materna.

Palabras Clave: Comprensión del discurso escrito, discurso académico, inglés para propósitos académicos.

* Trabajo enmarcado en el proyecto FondECYT 1060440, PUCV.

17-V-2007

Aceptado: 13-XI-2007
Correspondencia: Romualdo Ibáñez (romualdo.ibanez @ucv.cl). Tel.: (56-32) 227 3378. Instituto de Literatura y Ciencias del Lenguaje, Pontificia Universidad Católica de Valparaíso. Avenida Brasil 2830, piso 9, Valparaíso, Chile. 


\title{
Comprehension of academic texts written in English: Relation between level of comprehension achieved and variables involved
}

\begin{abstract}
Nowadays, it is considered essential for university students to have psycho-discursive skills that allow them comprehend disciplinary texts successfully. However, as English has become a lingua franca, not only in the fields of international trading and technology development, but also in research and education, in countries where English is not the mother tongue, university students must also develop the ability to comprehend disciplinary texts written in English. Approaching this phenomenon, in this investigation, we examined the process of comprehension carried out by university students when facing disciplinary texts written in English. The students $(n=112)$ belonged to the Industrial Chemistry program, at Pontificia Universidad Católica de Valparaíso, Chile. We focused on the level of comprehension these students achieved when facing a disciplinary text written in English and also on the way that level of comprehension was predicted by the participants' English proficiency level, their degree of insertion in the disciplinary community, and their reading skill. We carried out a correlational study, which showed that the relation between the level of comprehension the students achieved and each of the variables considered does not represent a high positive correlation. However, through the analysis we carried out, we could state that the variable that best predicts the level of comprehension of an academic text written in English corresponds to English proficiency level, followed by degree of insertion in the disciplinary community, and, at the end, reading skill.
\end{abstract}

Key Words: Written discourse comprehension, academia discourse, English for academic purpose.

\section{INTRODUCCIÓN}

Sin duda, en la actualidad, existe consenso entre profesionales y académicos acerca de la relevancia que la comprensión y, en definitiva, el aprendizaje a partir de textos escritos representa en cualquier proceso de enseñanza-aprendizaje. Su importancia se incrementa aún más en la educación terciaria, puesto que, de acuerdo a especialistas como Bhatia $(2002,2004)$ y Hyland (2004), la interacción exitosa con textos de la disciplina constituye una vía fundamental de acceso a la misma, no solo en términos de adquisición de conocimientos específicos, sino que también en lo concerniente a la integración definitiva de los individuos a sus grupos disciplinares. Estos planteamientos, indudablemente, enfatizan la necesidad de poseer habilidades psicodiscursivas propicias para desenvolverse con éxito en el ámbito académico.

Lamentablemente, investigaciones realizadas en América Latina y particularmente en Chile, como es el caso de los trabajos desarrollados por Parodi (2007a) y Peronard (2007), revelan las serias incapacidades de los alumnos de nivel terciario al enfrentar textos escritos correspondientes a su ámbito disciplinar. Estas carencias, en términos de habilidades de comprensión, por supuesto, representan un obstáculo mayor en el recorrido de acceso a la disciplina. La situación se complejiza aún más para estos estudiantes si se considera la condición actual del inglés como lengua franca (Flowerdew \& Peacock, 2001), lo que, por supuesto, transforma a esta lengua en herramienta indispensable para acceder al conocimiento especializado. 
Por esta razón, en Chile, desarrollar habilidades para comprender textos académicos escritos en lengua materna se transforma en una condición necesaria pero no suficiente, especialmente cuando se toma en consideración la cantidad de material escrito en inglés que los estudiantes universitarios deben enfrentar, ya sea a través de sus textos de estudios (manuales y textos disciplinares), artículos científicos o material publicado en Internet.

Si bien este panorama resulta inquietante, los avances logrados en investigaciones centradas en el lenguaje y en su uso en ámbitos especializados permiten visualizar nuevas formas de enfrentar las dificultades y necesidades antes mencionadas. Específicamente, desde el análisis del discurso y el Inglés para Propósitos Académicos (IPA) existen líneas de investigación que se han focalizado en la descripción de textos pertenecientes a diferentes registros y géneros de especialidad (Bhatia, 1993, 2004; Swales, 2001, 2004; Martin \& Rose, 2007). Del mismo modo, en lo que respecta a la comprensión de textos de especialidad, existen líneas de investigación que, desde la psicolingüística (Parodi, 2007a) y la psicología del discurso (McNamara \& Kintsch, 1996; McNamara, 2004; Kintsch, 1998, 2002), se han centrado en los procesos cognitivos involucrados en la comprensión y el aprendizaje a partir del texto. A través de la integración de los progresos realizados en estas áreas de investigación es posible abordar el estudio de la comprensión de textos académicos escritos en inglés.

Asumiendo la relevancia, así como también la complejidad de la problemática en cuestión, en esta investigación abordamos la comprensión de textos académicos escritos en inglés. Para ello, partimos de la premisa de que este tipo de procesos presenta características generales e involucra conocimientos y habilidades que lo asemejan a cualquier proceso de comprensión de textos escritos, pero, al mismo tiempo, ostenta características particulares que lo diferencian tanto de un proceso de comprensión de textos escritos en lengua materna, como de un proceso de comprensión de textos no especializados.

Entendemos que -en el escenario aquí descrito- un proceso de comprensión de textos académicos escritos en inglés se diferencia de un proceso de comprensión de textos escritos en lengua materna, en tanto el dominio de la segunda lengua, como variable lingüística, juega un rol fundamental (Alderson, 1984, 2000). Asimismo, entendemos que la comprensión de textos académicos se diferencia de un proceso de comprensión de textos no especializados, pues, mientras este último involucra, preferentemente habilidades, conocimientos lingüísticos y conocimientos de mundo asociados a una comunidad de habla, un proceso de comprensión de textos académicos involucra, además, habilidades, conocimientos lingüísticos y conocimientos disciplinares específicos, asociados a una comunidad discursiva.

Por ello, para desarrollar la investigación, utilizamos un sustento teórico que integra la investigación en el área de la comprensión del discurso escrito (van Dijk \& Kintsch, 1983; Kintsch, 1988, 1998; Parodi, 2007a), la investigación en el área de la lectura en segunda lengua (Alderson, 
1984, 2000; Koda, 2005), la investigación en el área del discurso académico (Parodi, 2007b) y la teoría del género (Martin, 1992; Swales, 1990, 2001, 2004; Bhatia, 1993, 2004).

\section{Una perspectiva teórica integrada}

En esta investigación, concebimos la comprensión del discurso escrito como un proceso cognitivo intencionado de alta complejidad, constituido por una serie de procesos psicodiscursivos, sustentados a su vez, en una variedad de procesamientos cognitivos de orden inferior (atención, percepción y memoria) y de orden superior (toma de decisiones, monitoreo, reflexión, entre otros). Adherimos, así, a la idea de que a partir de la interacción de la totalidad de los procesos involucrados se genera una representación mental de la situación descrita en el texto, en base a la información textual, así como a los conocimientos previos del individuo (van Dijk \& Kintsch, 1983; Kintsch, 1988; 1998; Ibáñez, 2007a).

Reconocemos, además, que los diversos procesos involucrados en el macroproceso de comprensión no funcionan en forma secuencial ni encapsulada, sino que de manera interactiva y, generalmente, simultánea (van Dijk \& Kintsch, 1983; Kintsch, 1988, 1998, 2002; van den Broek \& Gustafson, 1999). Por lo tanto, las respectivas habilidades necesarias, como la habilidad para generar inferencias o para utilizar adecuadamente el conocimiento previo involucrado pueden ser requeridas en diferentes niveles de procesamiento.

En este punto, uno de los aspectos que consideramos esencialmente relevantes es el uso del conocimiento previo, razón por la cual, entendemos que una mirada integral al fenómeno que abordamos debe incluir a los textos, sus características y, decididamente, al conocimiento que el lector y aprendiente posee al respecto. Esto pues, creemos que el uso estratégico de tal conocimiento (van Dijk \& Kintsch, 1983) determinará, en gran medida, el nivel de comprensión alcanzado.

Entendemos un texto como una actualización particular de una lengua. Así, cada texto que se produce tiene lugar en una situación comunicativa específica y en un contexto general, los que determinan su configuración lingüística y estructural, a partir de un propósito, un tema y un estilo. Denominamos al correlato lingüístico del contexto de situación como registro y al correlato lingüístico del contexto general como género (Martin, 1992).

Por esta misma razón, y desde una perspectiva que surge a partir de los planteamientos de Halliday, McIntosh y Strevens (1964), creemos que cuando se trata de la comprensión de textos académicos escritos, la relevancia asignada al dominio de la dimensión lingüística, no debe focalizarse solo en el sistema de la lengua en la cual el texto esté escrito, sino que también debe ampliarse al registro en cuestión y al género al cual el texto pertenece. Además, sostenemos que cuando se trata de procesos de comprensión de textos académicos escritos en una segunda 
lengua -como es en este caso el inglés- tal dominio de la dimensión lingüística involucra tanto a la lengua materna como a la segunda lengua. La relevancia de este dominio en ambas lenguas se basa en el supuesto de que el lector debiera ser capaz de transferir ciertas habilidades y conocimientos utilizados en un proceso de comprensión desarrollado en lengua materna a un proceso de comprensión en segunda lengua (Alderson, 1984; Carrell \& Grabe, 2002; Grabe \& Stoller, 2002).

De acuerdo a lo que hemos venido planteando, es relevante señalar que, en un proceso de comprensión de las características que hemos descrito, no creemos necesario establecer una distinción entre segunda lengua y lengua extranjera. Ello debido a que, el contexto de uso de la lengua no está constituido por la cultura de un país o un grupo de países que comparten una lengua, sino, por una disciplina, entendida como una cultura particular (Hyland, 2004). Por esta razón, en este tipo de procesos, más que un dominio general del inglés, creemos fundamental poseer un dominio de los géneros y del registro que realizan los discursos en inglés que circulan en un ámbito disciplinar particular. En esta línea argumental, creemos que tal dominio del registro y de los géneros particulares que circulan en una disciplina determinada estará directamente asociado con el grado de inserción disciplinar de un individuo (Ibáñez, 2007b).

\section{La investigación}

Para llevar a cabo nuestro estudio, nos situamos en el contexto disciplinar de la carrera de Química Industrial de la Pontificia Universidad Católica de Valparaíso, Chile y nos centramos, particularmente, en dos propósitos. En primer lugar, nos interesa conocer el nivel de comprensión que los alumnos logran al enfrentar textos de su disciplina, escritos en inglés. En segundo lugar, nos interesa indagar en la manera en que tal nivel de comprensión se relaciona con variables que identificamos como centrales para nuestra investigación, a saber, el nivel de dominio del inglés de los alumnos, su grado de inserción disciplinar y su habilidad para comprender textos escritos en lengua materna.

Para ello, llevamos a cabo un estudio de alcance correlacional, utilizando un diseño no experimental transeccional y que está guiado por las siguientes hipótesis:

- $\quad \mathbf{H}_{1}$ : Existirá una relación positiva considerable entre el nivel de comprensión alcanzado por los alumnos al enfrentar un texto académico escrito en inglés y su nivel de dominio del inglés.

- $\mathrm{H}_{2}$ : Existirá una relación positiva media entre el nivel de comprensión alcanzado por los alumnos al enfrentar un texto académico escrito en inglés y su grado de inserción disciplinar. 
- $\mathbf{H}_{3}$ : Existirá una relación positiva considerable entre el nivel de comprensión alcanzado por los alumnos al enfrentar un texto académico escrito en inglés y el nivel de comprensión alcanzado al enfrentar un texto académico escrito en español.

Para describir los grados de relación, en nuestras hipótesis, los valores serán medidos a partir de la aplicación del Coeficiente de Correlación de Pearson (Hatch \& Lazaraton, 1991; Hernández, Fernández \& Baptista, 2006) y del Gamma de Goodman y Kruskal (Conover, 1999), dependiendo de las necesidades y características del análisis.

\subsection{Los sujetos}

Nuestro estudio es de carácter censal, por lo que apuntamos a trabajar con la población completa, la que corresponde a la totalidad de los alumnos de la carrera de Química Industrial de la Pontificia Universidad Católica de Valparaíso. El detalle de esta información se presenta en la Tabla 1.

Tabla 1. Número de alumnos participantes y año de ingreso.

\begin{tabular}{|c|c|}
\hline Año de ingreso & Número de alumnos \\
\hline 2002 & 8 \\
\hline 2003 & 9 \\
\hline 2004 & 9 \\
\hline 2005 & 14 \\
\hline 2006 & 14 \\
\hline 2007 & 30 \\
\hline TOTAL DE ALUMNOS & 84 \\
\hline
\end{tabular}

\subsection{Construcción y selección de los instrumentos}

Para llevar a cabo la recolección de datos, utilizamos tres instrumentos: una prueba para medir la comprensión de un texto académico escrito en español; una prueba para medir la comprensión de un texto académico escrito en inglés; una prueba estandarizada para medir el dominio del inglés de los alumnos.

Antes de profundizar en la descripción de los instrumentos utilizados, es preciso señalar que concebimos la inserción disciplinar como el proceso de formación a través del cual los miembros de una determinada comunidad disciplinar desarrollan competencias y conocimientos especializados asociados a la disciplina, lo que les permite transformarse de novatos en expertos. Tales 
conocimientos y competencias, según Hyland (2004), son desarrollados a través de las prácticas discursivas propias y distintivas de cada comunidad. En este sentido, creemos que el grado de inserción disciplinar de los alumnos es un indicador certero de su conocimiento acerca de los géneros discursivos típicos que circulan en su comunidad y, por lo tanto, tendrá incidencia en los resultados de nuestra investigación. Además, entendemos que el grado de inserción estará determinado por el grado de participación y el tiempo de pertenencia en la comunidad. Por dicha razón, en esta investigación, distinguimos el grado de inserción disciplinar de los alumnos en base a su año de ingreso a la carrera.

\subsubsection{Primer instrumento: evaluación de la comprensión de texto académicos escritos en} español

El instrumento destinado a la evaluación de la comprensión del texto académico escrito en español fue seleccionado desde una investigación anterior (Parodi, 2007a), debido a su adecuación en términos teóricos y metodológicos. La concepción de comprensión que subyace al instrumento reconoce la generación de tres niveles de representación discursiva y dos planos estructurales. Nos referimos al código de superficie, a la base textual, al modelo de situación, a la microestructura y a la macroestructura del texto, respectivamente. Asimismo, tanto para alcanzar los niveles de representación, como para establecer los dos tipos de estructura señalados, se asume necesario llevar a cabo ciertos procesos psicodiscursivos indispensables, tales como la identificación de información explícita o literal, la generación de inferencias y la integración de la información en un significado coherente.

Por esta razón, este instrumento compuesto por catorce preguntas, evalúa tanto los niveles de representación alcanzados, los planos estructurales y los procesos psicodiscursivos involucrados. El puntaje asignado a las preguntas responde al rol que se le otorga a un determinado proceso psicodiscursivo dentro de una jerarquía de dificultad. Esto quiere decir que el instrumento pretende medir diferentes procesos, así como diferentes niveles de representación, todo ello con el fin de comprobar el efectivo nivel de comprensión profunda del texto.

De este modo, las preguntas de corte literal miden el reconocimiento de la información que es directamente derivable desde un fragmento específico del texto. La exigencia que representa este proceso es la menor dentro de la jerarquía de dificultad, puesto que a partir de él, solo es posible acceder al nivel de representación discursiva correspondiente al código de superficie. Por ello, a las preguntas literales se les otorgó un grado inferior de puntaje, es decir, cuentan con un peso reducido dentro del puntaje total general de evaluación en el instrumento.

A las preguntas que apuntan a evaluar la generación de inferencias locales de tipo léxica, correferencial y causa-consecuencia, se les asignó un mayor puntaje respecto del tipo de pregunta 
anterior, ya que los procesamientos involucrados implican un mayor grado de exigencia dentro de la jerarquía de dificultad. Esto debido a que entendemos que las inferencias locales contribuyen a la construcción de la microestructura del texto a partir de la vinculación de información explícita y no explícita en el texto.

Las preguntas denominadas de comprensión global apuntan a evaluar la construcción de la macroestructura del texto. La generación de esta estructura implica la articulación de la información, a partir de estrategias de integración, generalización o eliminación. Cabe señalar que este proceso, junto con la generación de la microestructura del texto, permite alcanzar el nivel correspondiente a la base textual. Por supuesto, esta clase de procesamiento implica un mayor grado de exigencia dentro de la jerarquía de dificultad, por lo que a este tipo de preguntas se le asignó un mayor puntaje.

La última pregunta, denominada de aplicación, apunta a la evaluación del modelo de situación. Este nivel de representación del discurso es considerado el más profundo en cuanto a los niveles de comprensión que se pueden alcanzar, ya que implica la integración del significado del texto con los conocimientos previos, generando una representación de la situación descrita en el texto. Alcanzar este nivel de comprensión, según Kintsch $(1998,2002)$, implica aprendizaje y, por lo tanto, la capacidad de utilizar dicho conocimiento nuevo en situaciones novedosas y diversas. Por esta razón, la pregunta que mide este nivel es la que ostenta el más alto puntaje.

El segundo aspecto involucrado en el diseño y construcción de este instrumento estuvo constituido por las variables del texto. El texto seleccionado corresponde a un segmento de un ejemplar del género manual y la temática aborda la medición de la presión atmosférica, centrándose, particularmente, en las cualidades del barómetro de Bourdon. Cabe señalar, además, que para la investigación original, el grado de especialización de este texto fue determinado a partir de la aplicación del Análisis Multidimensional (AMD) (Parodi, 2005), lo que permitió clasificar al texto como uno de alta densidad informativa. Según Parodi (2005), esto quiere decir que este texto, de aproximadamente mil palabras, ostenta un alto grado de especialidad, en cuanto a la temática, los rasgos lingüísticos que lo constituyen y, en definitiva, a su organización estructural.

Para determinar, no solo el grado de especialización del texto, sino su nivel de adecuación a la disciplina en cuestión, fue necesario someterlo al juicio de expertos en el área de la Química Industrial. De este modo, se determinó que tanto la temática abordada, como el género discursivo, en este caso, el manual, correspondían a la disciplina.

\subsubsection{Segundo instrumento: evaluación de la comprensión de texto académicos escritos en inglés}

El instrumento fue diseñado y construido en base al anteriormente utilizado para evaluar la 
comprensión del texto académico escrito en español. Por ello, está constituido por el mismo tipo y número de preguntas, las que, por supuesto, apuntan a la evaluación de los mismos procesos y niveles de comprensión que el instrumento anteriormente descrito. Cabe señalar que las preguntas fueron planteadas en español y los alumnos debieron contestar en el mismo idioma. Esto permitió evaluar lo que los alumnos comprendieron del texto, sin la interferencia de las posibles limitaciones de los alumnos en términos de su capacidad para expresarse en inglés en forma escrita.

Con el objetivo de establecer una validez de contenido, se llevó a cabo un proceso de triangulación por medio del juicio de ocho pares expertos. Los resultados de este paso metodológico indicaron que existía un acuerdo interpares sobre el $90 \%$ en cuanto al contenido y formato de la prueba. Además, y con el fin de probar el texto y las preguntas, este instrumento fue aplicado a una muestra exploratoria piloto compuesta por estudiantes en proceso de práctica y profesores de la carrera en cuestión. Por otra parte, aplicado el alfa de Cronbach, el instrumento presenta un coeficiente de confiabilidad de 0,77 , es decir, su confiabilidad es aceptable.

Otro aspecto central en el diseño y construcción de este instrumento estuvo constituido por las variables del texto. El texto seleccionado corresponde a un ejemplar del género manual y la temática aborda el proceso de reacción oxidación-reducción. La pertinencia, tanto del género discursivo, como del texto en particular y su temática fue determinada a partir del juicio de expertos en el área de la Química Industrial, quienes, no solo participaron en la validación del texto seleccionado, sino también en la selección del género, la temática y el texto mismo. Además, el grado de especialización del texto fue corroborado por los miembros competentes de la comunidad disciplinar, con un acuerdo de un $95 \%$.

\subsubsection{Tercer instrumento: evaluación del nivel de dominio del inglés}

El instrumento destinado a la evaluación del nivel de dominio del inglés corresponde al Quick Placement Test (QPT). Esta prueba ha sido diseñada por la Universidad de Cambridge, a través de su departamento de diseño y construcción de exámenes de inglés para hablantes de otras lenguas, ESOL Examinations (English for Speakers of Other Languages), como un instrumento que permite situar a los aprendientes de inglés como segunda lengua en diferentes niveles de acuerdo a su grado de dominio. Esto se presenta en la Tabla 2. 
Tabla 2. Puntaje y niveles QPT (ESOL Examinations, 2007).

\begin{tabular}{|c|c|}
\hline Puntaje QPT & Nivel QPT \\
\hline $0-15$ & Beginner \\
\hline $16-23$ & Elementary \\
\hline $24-30$ & Lower Intermediate \\
\hline $31-40$ & Upper Intermediate \\
\hline $48-54$ & Advanced \\
\hline $55-60$ & Very Advanced \\
\hline
\end{tabular}

Cada uno de los niveles que se especifican en la Tabla 2 corresponden, además, a niveles de dominio estandarizados, establecidos y descritos por ALTE (Asociación de Evaluadores de Lenguaje en Europa), una institución albergante y normadora de gran parte de las instituciones dedicadas al diseño y administración de pruebas de medición del dominio de segundas lenguas.

De acuerdo a ESOL Examinations (2007), esta prueba permite tomar decisiones respecto de la ubicación de los estudiantes en diferentes niveles o cursos en forma objetiva y eficiente. Asimismo, una de sus cualidades particulares es que puede ser aplicada a aprendientes de cualquier edad. Como otros tipos de instrumentos desarrollados por ESOL, esta prueba estandarizada consta de amplia validez y confiabilidad, por lo que es utilizada, tanto en Europa como en otros países del mundo.

\subsection{Aplicación de los instrumentos de medición}

La etapa de aplicación de instrumentos fue llevada a cabo durante cuarenta y cinco días. En este periodo de tiempo y, de acuerdo a lo planificado durante la etapa de preparación de la investigación, cada alumno enfrentó primero la prueba para evaluar la comprensión del texto académico escrito en inglés, luego la del texto académico escrito en español y, finalmente, la prueba para medir el nivel de dominio del inglés. Con esto se pretendió que la aplicación de la prueba de comprensión del texto académico escrito en inglés no estuviera contaminada por aplicaciones previas.

\section{Análisis de los resultados}

La descripción, análisis y discusión de los resultados se desarrolla en dos etapas. Esto pues, previo al análisis correlacional utilizado para corroborar nuestras hipótesis, consideramos necesario abordar, por separado, los resultados obtenidos a partir de la aplicación de los tres instrumentos. 


\subsection{Primera etapa: resultados obtenidos a partir de la aplicación de los instrumentos}

En esta primera etapa, y a partir de una estadística descriptiva simple, nos concentramos en los niveles de logro alcanzados por los participantes en la prueba de dominio del inglés, luego, en los alcanzados en la prueba de comprensión del texto académico escrito en español y, por último, en los correspondientes a la prueba de comprensión del texto académico escrito en inglés.

\subsubsection{Nivel de dominio del inglés}

La Tabla 3 presenta la distribución de los alumnos participantes de acuerdo al nivel de inglés en que se sitúan, así como también, el porcentaje que representa tal nivel en el total de alumnos. Además, la tabla muestra el puntaje correspondiente a cada nivel constituyente del instrumento utilizado (Quick Placement Test) y la forma en que dichos niveles se asocian a los niveles internacionales establecidos por ALTE.

Tabla 3. Resultados en términos de niveles.

\begin{tabular}{|c|c|c|c|c|}
\hline Nivel ALTE & $\begin{array}{c}\text { Puntaje por } \\
\text { nivel }\end{array}$ & Nivel QPT & $\begin{array}{c}\mathbf{N}^{\circ} \text { de alum- } \\
\text { nos }\end{array}$ & $\begin{array}{c}\text { Porcentaje de } \\
\text { alumnos por nivel }\end{array}$ \\
\hline Breakthrough & $0-15$ & Beginner & 33 & $39 \%$ \\
\hline 1 & $16-23$ & Elementary & 26 & $31 \%$ \\
\hline 2 & $24-30$ & Lower Intermediate & 21 & $25 \%$ \\
\hline 3 & $31-40$ & Upper Intermediate & 4 & $5 \%$ \\
\hline 4 & $48-54$ & Advanced & 0 & $0 \%$ \\
\hline 5 & $55-60$ & Very Advanced & 0 & $0 \%$ \\
\hline
\end{tabular}

Como se puede apreciar en la Tabla 3, los datos revelan una gran disparidad en términos de dominio y una clara concentración en los niveles más básicos, ya que los alumnos situados en los niveles Beginner, Elementary y Lower Intermediate corresponden al $95 \%$ del total de alumnos participantes. La gran cantidad de alumnos situados en los niveles Beginner y Elementary permite sostener que en la carrera de Química Industrial de la Pontificia Universidad Católica de Valparaíso, el 70\% de los alumnos posee un nivel bastante bajo de dominio del inglés. Esto se transforma en una situación preocupante, especialmente considerando que, de acuerdo a ALTE (2007), los individuos que alcanzan el nivel Beginner solo son capaces de utilizar el inglés en situaciones comunicativas simples, tales como comprender instrucciones básicas, participar en conversaciones factuales básicas en un tópico conocido, comprender información o instrucciones básicas, completar formularios básicos o escribir notas incluyendo día, fecha y lugar. Del mismo modo, según ALTE (2007), quienes alcanzan el nivel Elementary son capaces de expresar 
requerimientos u opiniones simples en un contexto familiar, comprender información explícita dentro de un área conocida, como textos de estudio simples o reportes en materias familiares.

\subsubsection{Nivel de comprensión de texto académico escrito en español}

El porcentaje total promedio alcanzado por los participantes en este instrumento correspondió a un $68,1 \%$. Este porcentaje de logro refleja un nivel de comprensión relativamente alto al enfrentar un texto académico escrito en español, puesto que, adhiriendo a investigaciones anteriores (Peronard, Gómez, Parodi \& Núñez, 1998), consideramos un $60 \%$ de logro como un nivel mínimo aceptable. No obstante, y como se sabe, a través del porcentaje de logro promedio total no es posible tener una idea del rendimiento individual de los participantes. Por ello, consideramos necesario presentar ciertos datos que permiten apreciar si dicho porcentaje realmente refleja la realidad de la gran mayoría de los alumnos participantes en nuestra investigación. A continuación, en la Tabla 4, presentamos la distribución de los alumnos en términos de sus porcentajes de logro y la equivalencia porcentual respecto del total de los alumnos participantes.

Tabla 4. Distribución de los alumnos en términos de porcentajes.

\begin{tabular}{|c|c|c|}
\hline $\begin{array}{c}\text { Rangos de porcentaje } \\
\text { de logro }\end{array}$ & $\begin{array}{c}\mathbf{N}^{\circ} \text { de alumnos } \\
\text { por rango }\end{array}$ & $\begin{array}{c}\text { Equivalencia porcentual respecto } \\
\text { del total de alumnos }\end{array}$ \\
\hline $0 \%-29 \%$ & 0 & $0 \%$ \\
\hline $30 \%-39 \%$ & 4 & $4,8 \%$ \\
\hline $40 \%-49 \%$ & 5 & $6,0 \%$ \\
\hline $50 \%-59 \%$ & 17 & $20,2 \%$ \\
\hline $60 \%-69 \%$ & 16 & $19,0 \%$ \\
\hline $70 \%-79 \%$ & 22 & $26,2 \%$ \\
\hline $80 \%-100 \%$ & 20 & $23,8 \%$ \\
\hline
\end{tabular}

Como se puede apreciar en la Tabla 4, solo el 10,8\% de los participantes obtuvo un porcentaje de logro inferior al 50\%. Del mismo modo, se puede observar que del $89 \%$ de los participantes supera el $50 \%$ de logro y más precisamente, los que alcanzan un nivel superior al mínimo aceptable, es decir, aquellos que superan el $60 \%$ de logro, corresponden al $69 \%$ del total de los alumnos. Estos datos permiten sostener que la mayoría de los participantes de nuestra investigación pueden ser considerados comprendedores, relativamente, competentes cuando se trata de enfrentar un texto de su disciplina escrito en español.

Descritos los resultados en términos de porcentajes promedio totales, procedemos a entregar los resultados, esta vez, por tipo de pregunta. Como señalamos en el apartado destinado a la descripción del instrumento, cada tipo de pregunta obedece a un grado diferente en la jerar- 
quía de dificultad (Parodi, 2007a), comenzando por las preguntas de tipo literal, en el extremo más simple, luego, las de tipo inferencial local, a continuación las enfocadas en la comprensión global y, por último, la de aplicación. En el Gráfico 1, presentamos los resultados de acuerdo a lo señalado.



Gráfico 1. Resultados por tipo de preguntas en español.

Como muestra el Grafico 1, los resultados reflejan un panorama parcialmente congruente con los sustentos teóricos de nuestra investigación. Ello, ya que, como habíamos previsto, se aprecia un descenso gradual en cuanto a los niveles de logro entre las preguntas de tipo literal, las de tipo inferencial local y las de comprensión global, debido, precisamente, al aumento de complejidad en cuanto a los procesos psicodiscursivos involucrados. Es importante destacar aquí que el bajo rendimiento en las preguntas de comprensión global revela la poca capacidad para construir una base textual del texto leído. No alcanzar este nivel de representación implica, desde nuestro sustento teórico (van Dijk \& Kintsch, 1983; Kintsch \& Rawson, 2005), una inhabilidad para integrar la información extraída del texto leído en un todo coherente. Sin embargo, no deja de llamar la atención lo que sucede con la pregunta de aplicación, en la cual los alumnos alcanzaron el segundo más alto nivel de logro.

De acuerdo a nuestro sustento teórico, las preguntas de aplicación deberían ser las que mayores complicaciones representan para los lectores, teniendo en cuenta la gran diversidad de procesos y recursos cognitivos involucrados. Por ello, los niveles de logro alcanzados en este tipo de pregunta particular constituyen un hecho altamente novedoso, ya que en investigaciones previas que han evaluado la comprensión desde este enfoque (Peronard et al., 1998; Parodi, 
2003, 2007a), el orden gradual de descenso en los niveles de logro ha obedecido a la jerarquía de complejidad planteada por la teoría.

Una posible explicación para el alto nivel de logro alcanzado en la pregunta de aplicación puede estar relacionado con el grado de inserción disciplinar y lo que esta variable constituye, no solo en términos de conocimiento teórico especializado, sino también, en lo que refiere a los conocimientos asociados a los géneros discursivos -en este caso el manual- característicos de una disciplina determinada (Bhatia, 2004). Según McNamara y Kintsch (1996), el uso estratégico del conocimiento previo puede suplir la escasez de significado provocada, en ciertas situaciones, por la incapacidad para generar inferencias o hacer uso de macroestrategias (van Dijk \& Kintsch, 1983), permitiendo, de esta forma, la construcción de un modelo de situación adecuado. En este sentido, en situaciones académicas propias de una disciplina particular, en que los textos escritos a enfrentar involucran temáticas, estructuras discursivas y rasgos lingüísticos recurrentes, el conocimiento acerca de estos aspectos, puede facilitar en gran medida el proceso de comprensión. Debido a que el grado de inserción disciplinar es considerado como una variable central de la investigación, ahondaremos en la descripción de su incidencia en el apartado correspondiente al análisis correlacional.

\subsubsection{Nivel de comprensión de texto académico escrito en inglés}

El porcentaje promedio alcanzado por los participantes al enfrentar el instrumento destinado a medir la comprensión de un texto académico escrito en inglés correspondió a un 47,7\%. Este porcentaje de logro refleja un nivel de comprensión definitivamente bajo, puesto que, como anteriormente señalamos, consideramos un $60 \%$ de logro como un nivel mínimo aceptable. No obstante ello, es preciso determinar si el porcentaje de logro promedio realmente refleja la realidad de la gran mayoría de los alumnos participantes. Por ello, a continuación, en la Tabla 5, presentamos la distribución de los alumnos en términos de sus porcentajes de logro y su equivalencia porcentual respecto del total de los alumnos participantes.

Tabla 5. Número de alumnos por rango de porcentaje de logro y su porcentaje de representatividad en el total de alumnos

\begin{tabular}{|c|c|c|}
\hline $\begin{array}{c}\text { Rangos de porcentaje } \\
\text { de logro }\end{array}$ & $\begin{array}{c}\mathbf{N}^{0} \text { de alumnos por } \\
\text { rango }\end{array}$ & $\begin{array}{c}\text { Equivalencia porcentual } \\
\text { respecto del total de alumnos }\end{array}$ \\
\hline $0 \%-29 \%$ & 20 & $23,8 \%$ \\
\hline $30 \%-39 \%$ & 12 & $14,3 \%$ \\
\hline $40 \%-49 \%$ & 14 & $16,7 \%$ \\
\hline $50 \%-59 \%$ & 14 & $16,7 \%$ \\
\hline $60 \%-69 \%$ & 13 & $15,5 \%$ \\
\hline $70 \%-79 \%$ & 6 & $7,1 \%$ \\
\hline $80 \%-100 \%$ & 5 & $6 \%$ \\
\hline
\end{tabular}


Como se aprecia en la Tabla 5, el 23,8\% de los participantes obtuvo un porcentaje de logro inferior al $30 \%$. Del mismo modo, se observa que el $54,8 \%$ de ellos no supera el $50 \%$ de logro y, más precisamente, los que alcanzan un nivel de comprensión considerado como el mínimo aceptable, es decir, aquellos que superan el $60 \%$ de logro, solo llegan al $28,6 \%$ del total de los alumnos. Estos datos permiten sostener que la mayoría de los participantes exhibe serios problemas de comprensión al enfrentar un texto de su disciplina escrito en inglés.

Al igual que hicimos con los resultados obtenidos en la medición de la comprensión del texto académico escrito en español, presentamos los resultados obtenidos de acuerdo a lo que hemos entendido como una jerarquía de dificultad (Parodi, 2007a) respecto de los procesos psicodiscursivos involucrados. Esto se muestra en el Gráfico 2.

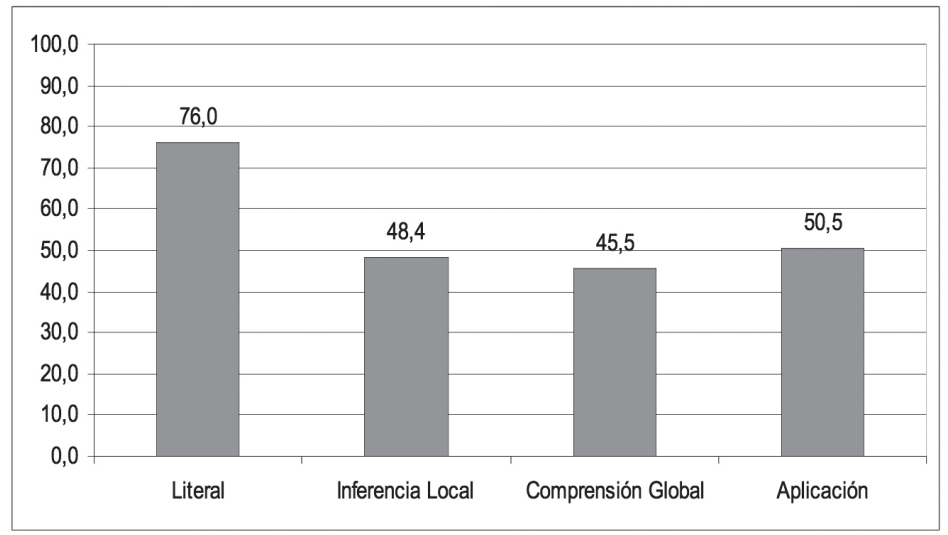

Gráfico 2. Resultados por tipo de preguntas en inglés

Como muestra el Grafico 2, y al igual que sucedió con la prueba de comprensión del texto académico escrito en español, los resultados reflejan un panorama parcialmente congruente con los sustentos teóricos de nuestra investigación. Ello se debe a que, si bien, es posible apreciar un descenso gradual en cuanto a los niveles de logro entre las preguntas de tipo literal, las de tipo inferencial local y las de comprensión global, la pregunta de aplicación refleja el segundo más alto nivel de logro. Es preciso mencionar, sin embargo, que las diferencias en los niveles de logro, en términos de porcentajes promedio por tipo de pregunta no son tan marcadas como en el caso de los resultados obtenidos en la prueba de comprensión del texto académico escrito en español. Esto se aprecia en las diferencias existentes entre las preguntas de tipo inferencial local, de comprensión global y de aplicación, en que los porcentajes promedio oscilan entre 45,5\% y 50,5\%. 
Se puede señalar, así, que en el proceso de comprensión desarrollado al enfrentar un texto académico escrito en inglés, los alumnos participantes enfrentaron mayores complicaciones al responder las preguntas de tipo inferencial local y las de comprensión global en comparación con los otros tipos de preguntas incluidos en el instrumento. En lo referente a los resultados obtenidos en las preguntas de tipo inferencial local, se puede observar que las deficiencias -ya evidenciadas a partir de la aplicación del QPT- en términos de conocimientos lexicogramaticales y semánticos afectaron directamente la construcción de la microestructura. Por otra parte, respecto de las preguntas de comprensión global, se podría inferir que las mayores dificultades de estos alumnos están relacionadas con ciertas habilidades que no dependen de una lengua particular, como son las asociadas a la construcción de la macroestructura del texto. Es pertinente recordar que los participantes de la investigación tampoco demostraron poseer dichas habilidades en el proceso desarrollado en lengua materna.

Así también, los resultados obtenidos reflejan que las complicaciones que podría representar un proceso de comprensión desarrollado en una segunda lengua y, a partir de un texto especializado, no se reflejan, mayormente, en las preguntas de tipo literal. Como señalamos anteriormente, estas solo implican el reconocimiento de información explícita en el texto, lo que, en el caso de un proceso de comprensión de textos académicos escritos en inglés, puede facilitarse a partir del uso estratégico de conocimientos disciplinares relacionados con el léxico especializado y con otros rasgos prototípicos de un género que circula en una determinada disciplina como es, en este caso, el manual.

Un hecho novedoso está constituido, nuevamente, por el nivel de logro alcanzado en la pregunta de aplicación, pues, aunque en forma leve, supera los niveles de logro obtenidos en preguntas que, supuestamente, apuntan a procesos psicodiscursivos de menor complejidad. Lo interesante de estos resultados radica en que, en esta situación, no solo se debe considerar la gran diversidad de procesamientos y recursos cognitivos implicados para alcanzar este nivel de comprensión, sino también las dificultades propias de llevar a cabo este proceso en una segunda lengua. Lo sucedido con esta pregunta, lleva a pensar que, al momento de generar un modelo de la situación descrita en el texto, la falta de un dominio de la segunda lengua en términos lexicogramaticales se ve compensada por otros tipos de conocimientos, en este caso, de tipo disciplinar. De esta manera, los conocimientos disciplinares asociados al género manual, se concretizan -debido a las características propias de la disciplina en cuestión- a partir de estrategias cognitivas, entre las que van Dijk y Kintsch (1983) destacan las de resolución de problemas. Desde esta perspectiva, la aparente contradicción con el sustento teórico no sería tal, puesto que se trataría de la integración de diversos tipos de conocimiento, a partir de estrategias cognitivas diversas para solucionar el problema planteado.

Este hallazgo nos lleva a reflexionar acerca de la manera en que concebimos la comprensión del 
discurso y, por supuesto, acerca de la forma en que la evaluamos, particularmente cuando se trata de discursos disciplinares. Creemos que en este tipo de situaciones, el grado de inserción disciplinar juega un rol central, debido, precisamente, a los conocimientos involucrados, entre los que se pueden señalar los de tipo discursivo y los asociados a las tareas y actividades típicas de la disciplina.

La primera etapa en el análisis de los resultados obtenidos en nuestra investigación ha tenido como propósito describir el comportamiento individual de las variables que, posteriormente, sometimos al análisis correlacional. Nos interesaba, precisamente, establecer un panorama que incluyera el nivel de dominio del inglés que los participantes de la investigación poseen, su capacidad para comprender un texto académico escrito en español y, por supuesto, el nivel de comprensión que ellos alcanzan al enfrentar un texto académico escrito en inglés. Habiendo realizado los análisis correspondientes a nuestro primer objetivo, procederemos al análisis correlacional.

\subsection{Análisis correlacional}

3.2.1. Relación entre el nivel de comprensión del texto académico escrito en inglés y el nivel de dominio del inglés

Para llevar a cabo el análisis, seguimos el orden establecido por el planteamiento de las hipótesis. Por ello, la primera relación que abordamos corresponde a la que hemos establecido entre la variable nivel de comprensión del texto académico escrito en inglés y la variable nivel de dominio del inglés. Más precisamente, nuestra hipótesis sostiene que existirá una relación positiva considerable $\left(r_{x y}>0,74\right.$ y $\left.<0,89\right)$ entre el nivel de comprensión alcanzado por los alumnos al enfrentar un texto académico escrito en inglés y su nivel de dominio del inglés.

Aplicada la prueba estadística para corroborar nuestra primera hipótesis, tenemos como resultado que existe un Gamma de 0,40 entre el nivel de comprensión alcanzado por los alumnos al enfrentar un texto académico escrito en inglés y su nivel de dominio del inglés. Esto equivale a una relación positiva débil entre las dos variables, por lo que nuestra hipótesis no ha sido corroborada totalmente. De acuerdo al Gamma obtenido, la posibilidad de que a medida que aumenta el puntaje alcanzado en la prueba de comprensión del texto académico escrito en inglés, los alumnos se sitúen en un nivel superior de dominio del inglés no es altamente probable.

3.2.2. Relación entre el nivel de comprensión del texto académico escrito en inglés y el grado de inserción disciplinar

La segunda relación que abordamos corresponde a la que hemos establecido entre la variable 
nivel de comprensión del texto académico escrito en inglés y la variable grado de inserción disciplinar. Nuestra segunda hipótesis sostiene que existirá una relación positiva media $\left(r_{x z}>0,49 y\right.$ $<0,75$ ) entre el nivel de comprensión alcanzado por los alumnos al enfrentar un texto académico escrito en inglés y su grado de inserción disciplinar.

Aplicada la prueba estadística para corroborar nuestra segunda hipótesis, tenemos como resultado que existe un Gamma de 0,21. Esto equivale a una relación positiva débil $\left(r_{x z}=0,21\right)$ entre las dos variables, por lo que podemos señalar que nuestra hipótesis ha sido corroborada solo parcialmente.

3.2.3. Relación entre el nivel de comprensión del texto académico escrito en inglés y el nivel de comprensión del texto académico escrito en español

La tercera relación corresponde a la que hemos establecido entre la variable nivel de comprensión del texto académico escrito en inglés y la variable nivel de comprensión del texto académico escrito en español. Más precisamente, nuestra hipótesis sostiene que existirá una relación positiva considerable $\left(r_{x w}>0,74 \mathrm{y}<0,89\right)$ entre el nivel de comprensión alcanzado por los alumnos al enfrentar un texto académico escrito en inglés y el nivel de comprensión alcanzado por los alumnos al enfrentar un texto académico escrito en español.

Aplicada la prueba estadística para corroborar nuestra tercera hipótesis, el coeficiente de correlación corresponde a 0,14 . Esto equivale a una relación positiva muy débil entre las dos variables, por lo que nuestra hipótesis ha sido corroborada solo parcialmente.

\subsection{Análisis por agrupamiento}

Los propósitos que guían esta investigación se focalizan, principalmente, en conocer y relacionar los resultados obtenidos por los individuos constituyentes de la población en estudio, no solo en términos generales, sino que también, y principalmente, en términos individuales. A partir de esta indagación, nos aproximamos a las posibles causas para los niveles de logro alcanzados por estos alumnos cuando enfrentan un texto académico escrito en inglés. Esto quiere decir que, como hemos venido argumentando, es preciso aproximarse a los resultados desde una mirada que nos permita visualizar la manera en que las variables involucradas se han configurado para determinar los niveles de logro obtenidos por los alumnos. Por ello, a través del Gráfico 3, mostramos que la escasez de una relación lineal se puede explicar a partir del surgimiento de lo que denominamos el clustering o agrupamiento de los datos. 


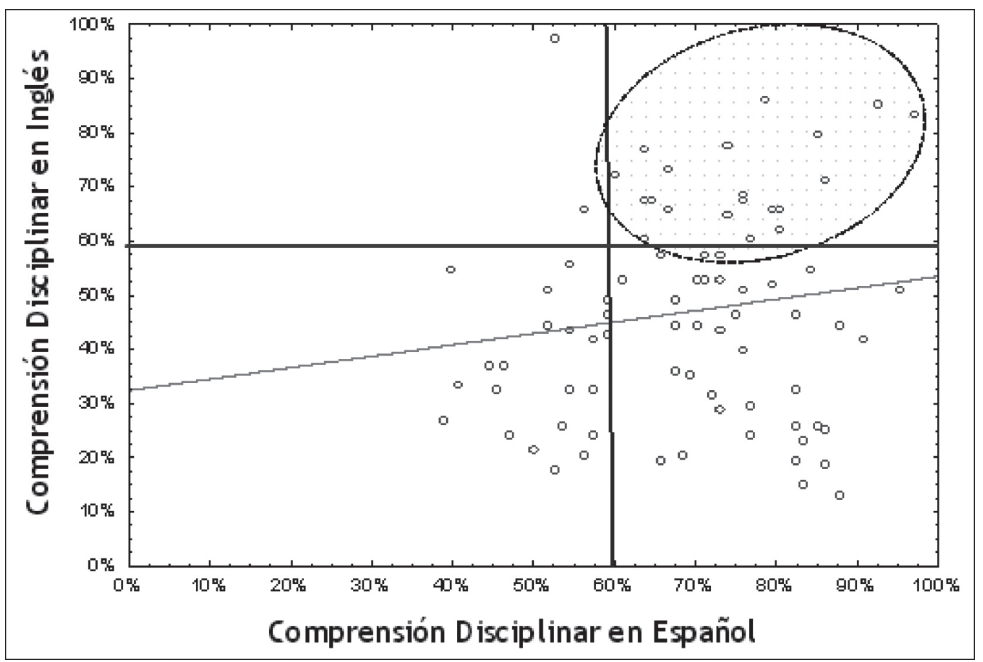

Gráfico 3. Diversas situaciones respecto de la relación de los resultados.

Para mostrar el agrupamiento de los datos, hemos dividido el gráfico en cuatro cuadrantes a partir de un criterio de división que se basa en un puntaje de corte del $60 \%$. Esto nos permite mostrar que la relación lineal esperada como resultado de nuestro análisis correlacional no se $\mathrm{da}$, debido a que los datos se distribuyen en lo que se puede interpretar como tres agrupaciones definidas. En primer lugar, en el cuadrante inferior izquierdo, es posible observar un número considerable de participantes cuyos resultados son bajos en ambas pruebas. Luego, en el cuadrante inferior derecho se puede apreciar a los sujetos que obtuvieron altos puntajes en la prueba de comprensión del texto académico escrito en español y bajos en la del texto académico escrito en inglés. Por último, en el cuadrante superior derecho, se puede apreciar a aquellos que obtuvieron puntajes altos en ambas pruebas. No obstante ello, es preciso destacar que, como muestra la línea de tendencia central, existe una inclinación, aunque mínima, al alza en los puntajes, lo que por supuesto corrobora el coeficiente de correlación obtenido a partir del estadístico aplicado.

La información presentada en el Gráfico 3, entrega más pistas acerca de los resultados obtenidos en el análisis realizado para corroborar nuestra tercera hipótesis. Así, se puede observar, en el cuadrante inferior izquierdo, que existen individuos, cuyos resultados fueron bajos, tanto en la prueba de comprensión del texto académico escrito en español como en la prueba de comprensión del texto académico escrito en inglés. Esto permite corroborar que existen individuos 
que, sencillamente, no poseen la capacidad para realizar un proceso de comprensión de manera adecuada, independiente de la lengua en que este se pretenda desarrollar e independiente del nivel de dominio del inglés que ellos posean. También, se puede observar en el cuadrante inferior derecho, que existe un gran número de individuos que sí posee la habilidad necesaria para comprender un texto en su lengua materna, pero que, al mismo tiempo, no es capaz de realizar dicho proceso en una segunda lengua. En este respecto, es necesario analizar el rol de su nivel de dominio del inglés, así como también, de su grado de inserción disciplinar, aspectos que abordaremos en conjunto más adelante. Por último, en el cuadrante superior derecho, se presenta una situación en que los individuos que evidenciaron ser capaces de desarrollar un proceso de comprensión en lengua materna adecuadamente, también fueron capaces de comprender un texto académico escrito en inglés. En este último caso, tampoco se debe restar importancia al rol de su grado de inserción disciplinar y su nivel de dominio del inglés.

Con el fin de profundizar en la forma en que las variables involucradas se configuran para determinar el nivel de logro alcanzado por los participantes de nuestra investigación, a continuación, en el Gráfico 4, presentamos la manera en que se relacionan el grado de inserción disciplinar, los puntajes obtenidos en ambas pruebas y el nivel de inglés de los alumnos. Para ello, en esta oportunidad, nos centramos en los puntajes obtenidos por los alumnos situados en el nivel de inglés correspondiente a Beginners. De acuerdo a las agrupaciones o clusters que se produzcan, podremos tener una idea acerca de la o las variables que tienen mayor influencia en el nivel de logro alcanzado por los alumnos.

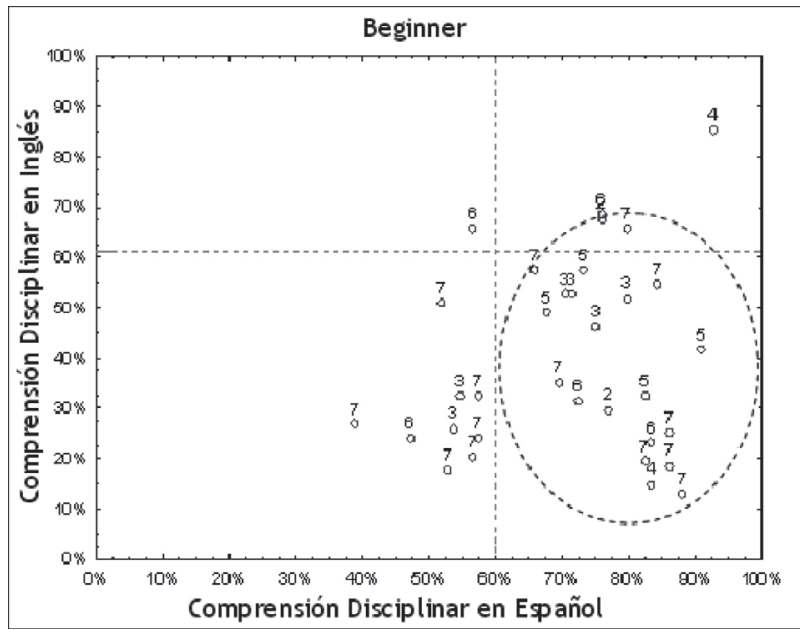

Gráfico 4. Relación entre las variables involucradas entre los alumnos con nivel de inglés Beginner. 
Al igual que hicimos anteriormente, dividimos el Gráfico 4 en cuatro cuadrantes a partir de un criterio de división, basado en un puntaje de corte del $60 \%$. Sin embargo, a diferencia de lo mostrado a través del Gráfico 3, en esta oportunidad, además de mostrar las agrupaciones que se forman, también identificamos el grado de inserción disciplinar de los participantes, centrándonos, como ya anticipamos, en los alumnos con nivel de dominio del inglés correspondiente a Beginners. A partir de esta mirada, podemos observar que en el cuadrante inferior izquierdo, se encuentra un grupo no menor de alumnos con año de ingreso 2003 y 2007 . Luego, en el cuadrante inferior derecho se puede apreciar un grupo mayor con años de ingreso desde 2002 hasta 2007. Por último, en el cuadrante superior derecho, se puede apreciar una extremada escasa presencia de alumnos, los que presentan año de ingreso 2004, 2006 y 2007.

La gráfica refleja que, en el caso de los alumnos que se sitúan en el cuadrante inferior izquierdo, aparte de su bajo nivel de inglés, tampoco son capaces de comprender un texto en su lengua materna, por lo que, independiente de su grado de inserción disciplinar, no fueron capaces de comprender el texto académico escrito en inglés. Por otra parte, en el cuadrante inferior derecho, podemos observar que los alumnos capaces de comprender un texto académico en su lengua materna, independiente de su grado de inserción disciplinar, no fueron capaces de utilizar su habilidad para, de este modo, comprender el texto académico escrito en inglés. Esto, debido, presumiblemente, a su bajo nivel de dominio del inglés. No obstante, y para asegurarnos de lo aquí planteado, a continuación presentamos, a través del Gráfico 5, el mismo tipo de comparación, pero esta vez, centrándonos en los alumnos con un nivel superior de dominio del inglés, es decir, los ubicados en los niveles Lower Intermediate y Upper Intermediate.

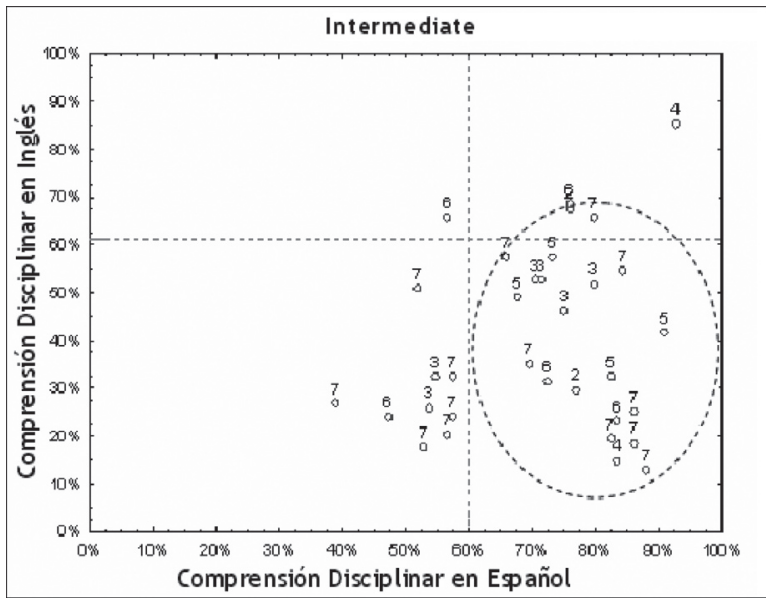

Gráfico 5. Relación entre las variables involucradas entre los alumnos con nivel de inglés Intermediate. 
A partir de esta segunda mirada, podemos observar, en primer lugar, que los puntajes obtenidos en la prueba de comprensión del texto académico escrito en inglés son definitivamente más altos que los presentados en el caso anterior. Luego, en una mirada enfocada en los cuadrantes, es posible advertir que en el cuadrante inferior izquierdo, se encuentra un grupo menor de alumnos con año de ingreso 2004, 2005 y 2007. En el cuadrante inferior derecho se puede apreciar un grupo no menor con años de ingreso 2006 y 2007. Por último, en el cuadrante superior derecho, también se puede apreciar un grupo no menor de alumnos, los que presentan año de ingreso desde 2003 hasta 2007.

Este análisis por agrupamiento corrobora los resultados obtenidos a partir del análisis correlacional respecto del rol relevante del nivel de dominio del inglés, por sobre el rol de la habilidad para comprender un texto escrito en lengua materna y del grado de inserción disciplinar cuando se trata de la comprensión de un texto académico escrito en inglés. Es interesante señalar que esta variable es la que determina el comportamiento de las demás, en tanto un umbral lingüístico es necesario para transferir la habilidad utilizada para comprender textos en lengua materna a una situación en que el texto a comprender está escrito en una segunda lengua. No obstante ello, no deja de llamar la atención lo sucedido con aquellos alumnos que se sitúan en el cuadrante inferior derecho. Esto pues, si bien, poseen un nivel de dominio del inglés que permitió a otros de sus compañeros utilizar su habilidad para comprender textos en lengua materna en el proceso desarrollado en segunda lengua, ellos no lo lograron. Al parecer, y poniendo atención a sus años de ingreso (2006 y 2007), el insuficiente grado de inserción disciplinar, independiente de su nivel de inglés y sus habilidades de comprensión, no les permitió alcanzar un mayor nivel de logro.

\section{CONCLUSIONES}

A partir de los datos obtenidos, es posible concluir que los niveles de logro alcanzados por los alumnos participantes de la investigación, tanto en la prueba de dominio del inglés como en la prueba de comprensión del texto académico escrito en inglés son, definitivamente, bajos. Esta situación se torna preocupante si se considera que estos alumnos deben enfrentar textos académicos escritos en inglés en la mayoría de sus asignaturas y durante los cuatro años que contempla su programa de estudios.

Por otra parte, los análisis realizados nos permiten sostener que la variable más determinante del nivel de comprensión del texto académico escrito en inglés corresponde a la variable nivel de dominio del inglés, seguida de la variable inserción disciplinar y, por último, a la variable habilidad de comprensión en lengua materna. Cabe señalar, que si bien, el análisis correlacional demostró la existencia de una relación positiva en todos los casos estudiados, en las tres relaciones que se plantearon en la investigación, el nivel de correlación fue bajo. 
Así también, y debido a los resultados obtenidos en el análisis por agrupamiento, podemos afirmar que las variables involucradas se comportan de forma diferente en distintas situaciones. En el caso de la habilidad para comprender textos en lengua materna, esta solo puede ser transferida si el lector posee un nivel umbral de dominio del inglés. No obstante ello, tal nivel de dominio presenta diferentes grados de incidencia dependiendo del proceso psicodiscursivo involucrado, lo que se puede apreciar en los resultado obtenidos por tipo de pregunta. Al respecto, cabe señalar que lo aquí planteado fue difícil de detectar debido, precisamente, al bajo nivel de dominio del inglés de los alumnos. Por último, cuando se trata del grado de inserción disciplinar, esta variable solo presenta una incidencia directa en casos de niveles extremadamente bajos, no permitiendo la comprensión; esto independiente de la habilidad para comprender textos en lengua materna y del nivel de inglés de los alumnos.

Un aspecto que nos llamó poderosamente la atención está relacionado con los resultados obtenidos en las preguntas de aplicación, lo que nos lleva a reflexionar acerca de la manera en que concebimos y evaluamos la comprensión del discurso escrito, especialmente, cuando se trata de la comprensión de textos especializados. Esto pues, los niveles de logro alcanzados por algunos de los participantes de nuestra investigación en este tipo de preguntas de aplicación indican que ellos, supuestamente, fueron capaces de construir un modelo adecuado de la situación descrita en el texto, ya que demostraron poder aplicar los conocimientos extraídos desde el texto que leyeron a una situación nueva. No obstante, sus resultados en las preguntas inferenciales locales y de comprensión global indican que ellos no lograron generar una base textual del texto leído. Tal situación, nos lleva a plantear que estos alumnos habrían logrado alcanzar el tercer nivel de representación a partir de la utilización de estrategias cognitivas generales, directamente relacionadas con la disciplina y, en este sentido, asociadas a la resolución de problemas. Por supuesto, lo anterior nos lleva a inferir que el grado de inserción disciplinar y con esto, los conocimientos asociados al género -en este caso manual- tendrán un rol determinante cuando se trata de enfrentar preguntas de aplicación.

De acuerdo a lo aquí expuesto, parece pertinente preguntarse qué se debe entender por comprensión profunda de un texto especializado: (a) ¿el proceso desarrollado por un alumno capaz de realizar inferencias locales, así como también capaz de utilizar estrategias macroestructurales que le permitan alcanzar una base textual? o (b) ¿el proceso desarrollado por un alumno que logra alcanzar un modelo de situación en base, principalmente, a sus conocimientos disciplinares, entre los que se incluyen los de tipo discursivo? En el primer caso, de acuerdo a $\mathrm{MCNa}$ mara y Kintsch (1996) y a Kintsch $(1988,1998)$, el alumno sería capaz de extraer el significado global del texto e incluso de resumirlo; en el segundo, sería capaz de integrar la información a su conocimiento previo y aplicarla a situaciones nuevas, como queda en evidencia a partir de los resultados obtenidos en nuestra investigación. En este escenario, es importante tener en cuenta tanto los propósitos, como también, las características de los géneros que circulan 
en ámbitos de especialidad, puesto, que en muchos casos, estos describen procesos y plantean problemas, como es el caso de los manuales de Química utilizados en nuestra investigación. Por ello, aparentemente, los alumnos, más que, por ejemplo, extraer el significado global del texto leído, deben utilizar la información allí presentada para solucionar problemas concretos de su disciplina.

La investigación realizada nos permitió obtener datos relevantes acerca de la relación entre el nivel de comprensión de textos académicos escritos en inglés y las variables que hemos entendido como determinantes del nivel de logro alcanzado, especialmente, en lo concerniente al grado de inserción disciplinar, por ser este un factor fundamental y, sin embargo, pocas veces considerado cuando se evalúa un proceso de comprensión con estas características.

Del mismo modo, los resultados obtenidos nos motivan a continuar estudiando el fenómeno, pero, esta vez, desde una mirada más compleja, sin limitar el objetivo del estudio a la forma en que las variables determinan el nivel de comprensión de un texto académico escrito en inglés. Creemos que un siguiente paso contempla ampliar el foco de atención hacia otros ejes. Por un lado, es necesario avanzar hacia la descripción profunda de la interacción entre la totalidad de las variables involucradas y concentrarse en esta relación sinérgica, debido, precisamente, a su trascendencia no solo en el desempeño académico, sino que también, en el profesional. Por otro, creemos que es interesante ampliar la mirada disciplinar y comparar la forma en que estas y otras variables se relacionan a través de diferentes disciplinas. Lo aquí planteado se grafica en la Figura 1.

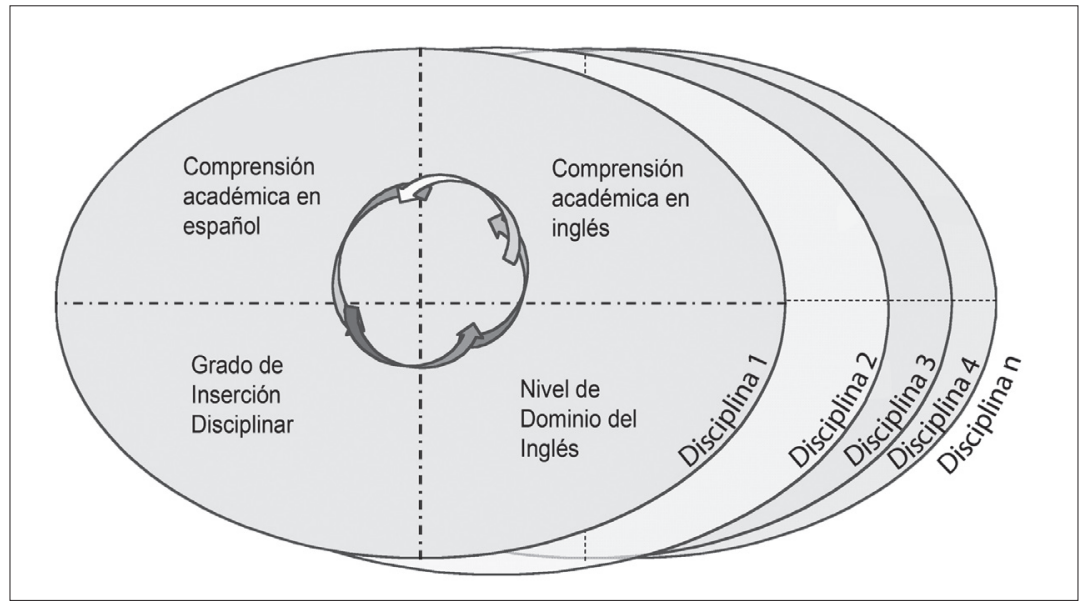

Figura 1. Variables involucradas en el perfil multidimensional en diversas disciplinas. 
Desde esta concepción del fenómeno, la cual se presenta en la Figura 1, podríamos construir un perfil multidimensional del procesamiento del lenguaje escrito en español y en inglés y en distintos ámbitos disciplinares. Esta mirada compleja desde diversas habilidades y conocimientos nos permitiría describir el modo en que estas variables interactúan y el peso relativo que cada una de ellas representa dentro de la formación universitaria especializada en áreas disciplinares epistémicamente diferentes.

\section{REFERENCIAS BIBLIOGRÁFICAS}

Alderson, J. (1984). Reading in a foreign language: A reading problem or a language problem? En J. Alderson \& A. Urquhart (Eds.), Reading in a foreign language (pp. 1-24). London: Longman.

Alderson, J. (2000). Assessing reading. Cambridge: Cambridge University Press.

Association of Languaje Testers in Europe (ALTE) (2007) [en línea]. Disponible en: http://www.alte. org/furtherinfo/framework_english.pdf

Bhatia, V. (1993). Analysing genre. Language use in professional settings. London: Longman.

Bhatia, V. (2002). A generic view of academic discourse. En J. Flowerdew (Ed.), Academic discourse (pp. 21-39). Cambridge: Cambridge University Press.

Bhatia, V. (2004). Worlds of written discourse. A genre based view. Sydney: Continuun.

Biber, D. (1988). Variation across speech and writing. Cambridge: Cambridge University Press.

Carrell, P. \& Grabe, W. (2002). Reading. En N. Schmitt (Ed.), An introduction to applied linguistics. (pp. 233-250). London: Arnold.

Conover, W. (1999). Practical nonparametric statistics. New York: John Wiley \& Sons.

Flowerdew, J. \& Peacock, M. (2001). Issues in EAP: A preliminary perspective. En J. Flowerdew \& M. Peacock (Eds.), Research perspectives on English for academic purposes (pp. 315-359). Cambridge: Cambridge University Press.

Grabe, W. \& Stoller, F. (2002). Teaching and researching reading. London: Pearson.

Halliday, M., McIntosh, A. \& Strevens, P. (1964). The linguistic science and language teaching. London: Longman.

Hatch, E. \& Lazaraton, N. (1991). The research manual. Design and statistics for applied linguistics. Massachusetts: Heinle \& Heinle Publishers.

Hernández, R., Fernández, C. \& Baptista, P. (2006). Metodología de la investigación. Santiago: McGraw-Hill.

Hyland, K. (2004). Disciplinary discourses. Social interactions in academic writing. London: Longman.

Ibáñez, R. (2007a). Cognición y comprensión. Una aproximación histórica y crítica al trabajo investigativo de Rolf Zwaan. Revista Signos. Estudios de Lingüística, 40(63), 81-100. 
Ibáñez, R. (2007b). Comprensión de textos disciplinares escritos en inglés. RLA, 45, 67-85.

Kintsch, W. (1988). The role of knowledge in discourse comprehension: A construction-integration model. Psychological Review, 95, 163-182

Kintsch, W. (1998). Comprehension: A paradigm for cognition. New York: Cambridge Univesity Press.

Kintsch, W. (2002). On the notions of theme and topic in psychological process models of text comprehension. En M. Louwerse \& W. van Peer (Eds.), Thematics: Interdisciplinary studies (pp. 151-170). Amsterdam: Benjamins.

Kintsch, W. \& Rawson, K. (2005). Comprehension. En M. Snowling \& CH. Hume (Eds.), The science of reading. A handbook (pp. 209-226). Victoria: Blackwell.

Koda, K. (2005). Insights into second language reading. A cross-linguistic approach. Cambridge: Cambridge University Press.

Martin, J. (1992). English text. System and structure. Philadelphia: Benjamins.

Martin, J. \& Rose, D. (2007). Genre relations. Mapping culture. London: Equinox.

McNamara, D. (2004). Aprender del texto: Efectos de la estructura textual y las estrategias del lector. Revista Signos. Estudios de Lingüística, 37(55), 19-30.

McNamara, D. \& Kintsch, W. (1996). Learning from texts: Effects of prior knowledge and text coherence. Discourse Processes, 22, 247-288.

Parodi, G. (2003). Relaciones entre lectura y escritura: Una perspectiva cognitiva discursiva. Valparaíso: Ediciones Universitarias de Valparaíso.

Parodi, G. (2005). Lingüística de corpus y análisis multidimensional: Exploración de la variación en el corpus PUCV-2003. En G. Parodi (Ed.), Discurso Especializado e Instituciones Formadoras (pp. 83-126). Valparaíso: Ediciones Universitarias de Valparaíso.

Parodi, G. (2007a). Comprensión y aprendizaje a partir del discurso especializado escrito: Teoría y empiria. En G. Parodi (Ed.), Lingüística de corpus y discursos especializados: Puntos de mira (pp. 223-258). Valparaíso: Ediciones Universitarias de Valparaíso.

Parodi, G. (2007b). El discurso especializado escrito en el ámbito universitario y profesional: Constitución de un corpus de estudio Revista Signos. Estudios de Lingüística, 40(63) 147-178.

Peronard, M. (2007). Lectura en papel y en pantalla de computador. Revista Signos. Estudios de Lingüística, 40(63), 179-195.

Peronard, M., Gómez, L., Parodi, G. \& Núñez, P. (1998). Comprensión de textos escritos: De la teoría a la sala de clases. Santiago: Andrés Bello.

Swales, J. (1990) Genre analysis: English in academic and research settings. Cambridge: Cambridge University Press.

Swales, J. (2001). EAP-related linguistic research: An intellectual history. En J. Flowerdew \& M. Peacock (Eds.), Research perspectives on English for academic purposes (pp. 42-54). Cambridge: Cambridge University Press. 
Swales, J. (2004). Research genre. Exploration and applications. Cambridge: Cambridge University Press.

van Dijk, T. \& Kintsch, W. (1983). Strategies of discourse comprehension. New York: Academic Press. van den Broek, P. \& Gustafson, M. (1999). Comprehension and memory for texts: Three generations of reading research. En S. Goldman, A. Graesser \& P. van den Broek (Eds.), Narrative comprehension, causality and coherence. Essays in honor of Tom Trabasso (pp. 15-34). Hillsdale, NJ: Erlbaum. 